\title{
The Anatomical Relationship of Inferior Thyroid Artery and Recurrent Laryngeal Nerve: A Review of the Literature and Its Clinical Importance
}

\author{
George Noussios $^{\mathrm{a}}$, Iosif Chatzis ${ }^{\mathrm{b}}$, Sergios Konstantinidis ${ }^{\mathrm{b}}$, Eva Filo ${ }^{\mathrm{b}}$, Antigone Spyrou ${ }^{\mathrm{b}}$, \\ George Karavasilis ${ }^{c}$, Anastasios Katsourakis ${ }^{\mathrm{b}, \mathrm{d}}$
}

\begin{abstract}
Background: Thyroidectomy is a quite common daily operation in general surgery. However, the anatomical structures of the region, mainly the relationship of recurrent laryngeal nerve (RLN) and inferior thyroid artery (ITA) makes the procedure challenging. The current review of the literature aims to report the anatomical variations of this relationship.

Methods: The preferred reporting items for systemic reviews and meta-analyses (PRISMA) guidelines were used for the systematic review of the articles found after an extensive research through PubMed, Science Direct, EMBASE and Web of Science. A total of 16 studies were included for the statistical analysis.
\end{abstract}

Results: The results showed that the most common type of RLN was posterior to the ITA. However, according to Higgins I ${ }^{2}$ statistics the heterogeneity of the studies was quite high.

Conclusions: The relationship between the RLN and the ITA is quite variable. Anatomical knowledge of the region is vital for attempting to eliminate the risk of injuring the nerve during thyroidectomy.

Keywords: Inferior thyroid artery; Recurrent laryngeal nerve; Anatomy; Thyroidectomy

\section{Introduction}

The anatomical relationship between the recurrent laryngeal

Manuscript submitted July 23, 2020, accepted August 4, 2020

Published online September 21, 2020

aDepartment of Physical Education and Sports Sciences of Serres, Aristotle University of Thessaloniki, Thessaloniki, Greece

bDepartment of General Surgery, AgiosDimitrios General Hospital, Thessaloniki, Greece

'International Hellenic University, Serres, Greece

${ }^{\mathrm{d} C o r r e s p o n d i n g ~ A u t h o r: ~ A n a s t a s i o s ~ K a t s o u r a k i s, ~ D e p a r t m e n t ~ o f ~ G e n e r a l ~ S u r-~}$ gery, AgiosDimitrios General Hospital, Thessaloniki, Greece.

Email: tasoskatsourakis@hotmail.com

doi: https://doi.org/10.14740/jocmr4296 nerve (RLN) and the inferior thyroid artery (ITA) is volatile (Fig. 1). The ITA usually arises from the thyrocervical trunk and supplies the thyroid gland, while the RLN, which is a mixed nerve, is one of the branches of the vagus nerve. The RLN most commonly runs anterior to the ITA on the right side and posterior to the ITA on the left side. Sporadically, the RLN passes between the branches of the ITA.

During thyroidectomy, the RLN is at risk of iatrogenic injury. This is one of the major complications of thyroidectomy surgery, occurring in $2-5 \%$ of patients without nerve variations, and it results in voice and swallowing problems. To decrease these complications, a precise surgical technique is necessary to identify the ITA, which is the landmark for recognizing the RLN. The relationship between the artery and the nerve was studied during the past century, and several classification systems were developed. The aim of our study is to provide an evidence-based assessment of the anatomical relationship between the ITA and the RLN to reduce the incidence of iatrogenic injuries and postoperative complications of thyroid surgery.

\section{Materials and Methods}

\section{Search method}

An extensive research was performed of the following databases, which met the inclusion criteria for the analysis: PubMed, Science Direct, EMBASE, and Web of Science. The search terms used were "RLN", "ITA", "thyroid surgery", "complications", "anatomy", and "variations". For the analysis, we included articles published in English from 2005 to 2019. No review protocol existed. Case reports, letters to the editors, and conference abstracts were excluded from the study, and only original articles were used. The analysis was performed according to the preferred reporting items for systemic reviews and meta-analyses (PRISMA) guidelines and conducted independently by two separate reviewers, who were responsible for the data extraction. The data included the study, type of patients (cadavers or perioperative finding), and number of RLNs and their relationship to the ITA. Further statistical consultation was conducted if there was any disparity regarding the data. 


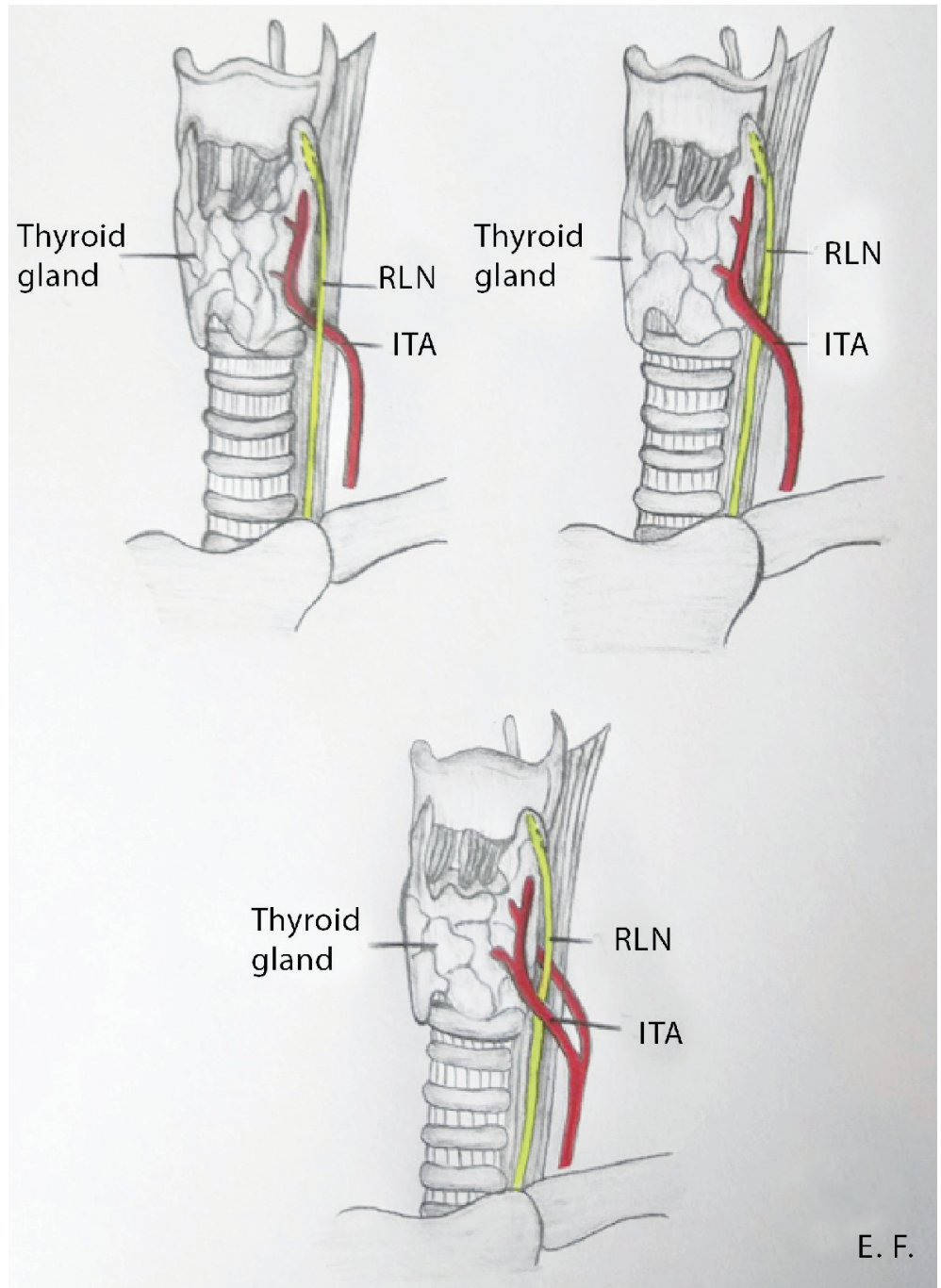

Figure 1. The anatomical relationships between the RLN and the ITA. RLN: recurrent laryngeal nerve; ITA: inferior thyroid artery.

\section{Statistical analysis}

The random effects model MetaXL 5.3 (freely available software for meta-analysis in Microsoft Excel) was used to examine the heterogeneity of the pooled data. The pooled prevalence and Cochran's Q test were estimated to identify heterogeneity among the studies. If the confidence intervals (CIs) of the pooled prevalence estimate overlapped poorly, this was strong evidence of statistical heterogeneity. The $\mathrm{I}^{2}$ statistics (Higgins statistics) were calculated to describe the percentage of the variability in effect estimates that was due to heterogeneity rather than sampling error.

\section{Results}

An initial search of the databases identified a total of 578 records; 511 articles did not meet the inclusion criteria, and 38 were duplicates. Twenty-nine full-text articles were assessed for eligibility. Of these, 13 articles were excluded due to in- complete data and statistical issues, and finally, 16 articles were used in the qualitative and quantitative analysis (Fig. 2). A total of 12 studies were performed on cadavers, and the other four were from intraoperative findings (Table 1 [1-16]).

A total of 4,198 RLNs (2,099 patients) were assessed for the statistical analysis. The most common type of RLN was posterior to the ITA, with a prevalence estimate of $52.08 \%$ ( $95 \%$ CI: 46.5 - 57.6), while the second most common was anterior to the ITA, with a pooled prevalence of $23.91 \%$ (95\% CI: 16.8 - 31.7). The least common type was between the branches of the ITA, with a prevalence estimate of $18.94 \%$ (95\% CI: 13.3 - 25.6). In addition, there were differences between the left and right sides, with the posterior type being the most common, occurring in $35.73 \%$ ( $95 \%$ CI: 32.57 - 38.95) of the nerves on the left side and $20.78 \%$ (95\% CI: $14.2-28.1$ ) of those on the right one (Table 2). The data from the included studies were not statistically sufficient in order to conclude any sex or race related difference in the local anatomy of ITA and the RLN.

In all the above cases, according to the Higgins $\mathrm{I}^{2}$ statistics, the heterogeneity was quite high, except in the case of the rela- 


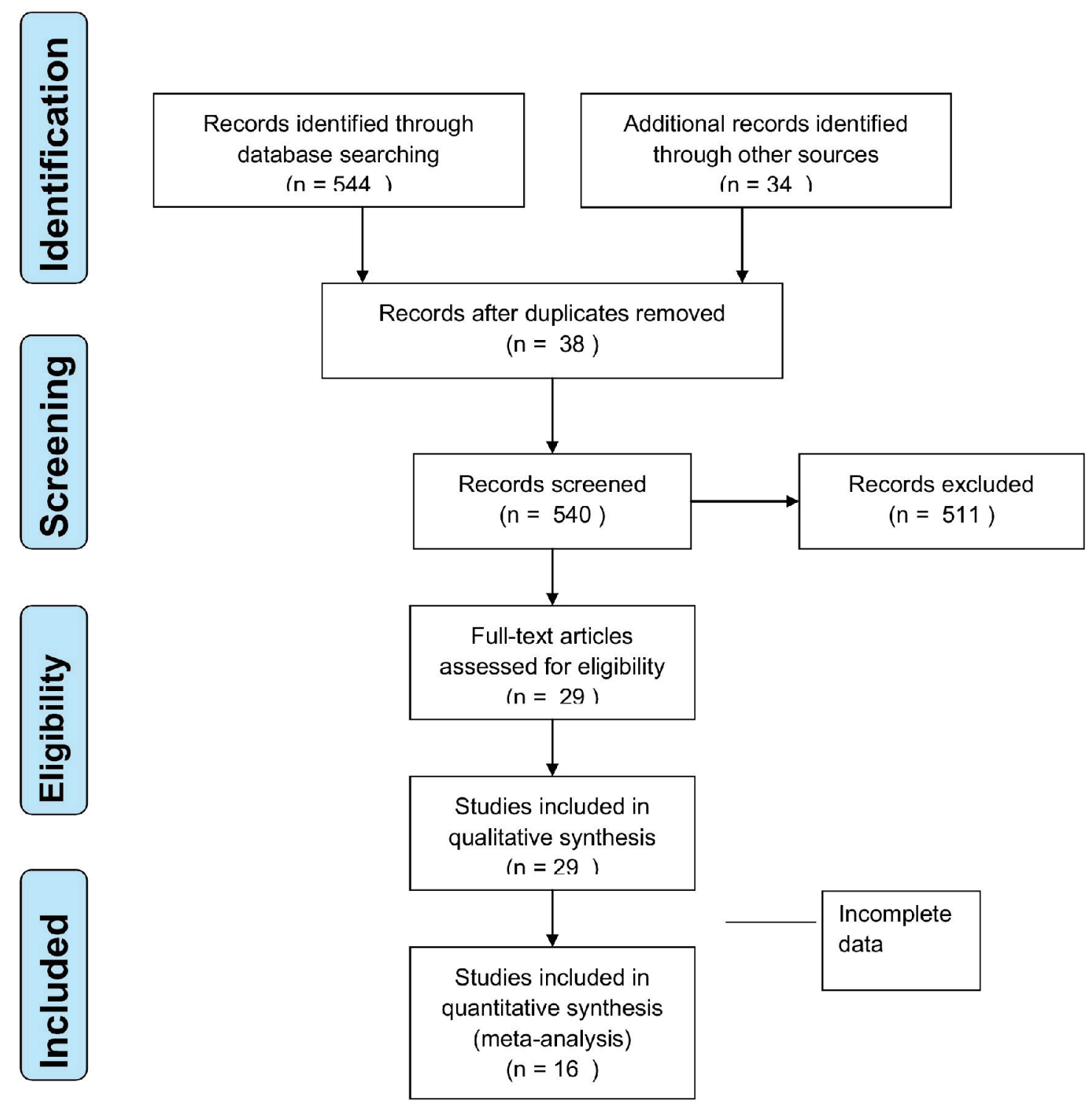

Figure 2. Flow diagram according to PRISMA guidelines. PRISMA: the preferred reporting items for systemic reviews and metaanalyses.

tionship of the RLN running posterior to the ITA at the left side. Despite the relatively large sample size more studies should be conducted to achieve more reliable conclusions (Figs. 3 - 6).

\section{Discussion}

The first well-described enlargement of the thyroid gland goes back to the ages of the Roman Empire; later, a detailed explanation of the thyroid anatomy was given in the 16th century AD by Leonardo da Vinci [17]. Surgery for thyroid diseases has evolved over the years; however, despite the vast decrease in morbidity, this is still an issue, and injury of the RLN can have a detrimental impact on patients' quality of life and cause psychosocial distress [18].
According to several studies, injury of RLN during thyroidectomy varies from $1.5 \%$ to $14 \%$ [19-21]. Recovery takes place within 1 year in more than $80 \%$ of cases [22]. The most common cause of litigation related to performed surgical operations worldwide is iatrogenic vocal cord palsy [23]. The main mechanisms of damaging the RLN during thyroidectomy are pressure, laceration, thermal damage, division, ligation, ischemia, and manipulation [24-26]. Additional lack of surgical plan during dissection, caused by fibrosis and scar tissue resulting from previous interventions, is a well-known reason for iatrogenic damage of the RLN. The result is that $12.5 \%$ of patients suffer from temporary vocal cord paralysis, and unfortunately, 3.8\% will experience permanent paralysis [27].

Fowler [28], and later, Skandalakis et al [29] concluded that injury to the RLN is more common when the nerve lies 
Table 1. The Included Studies and the Prevalence of Each Type of RLN to ITA Relationship

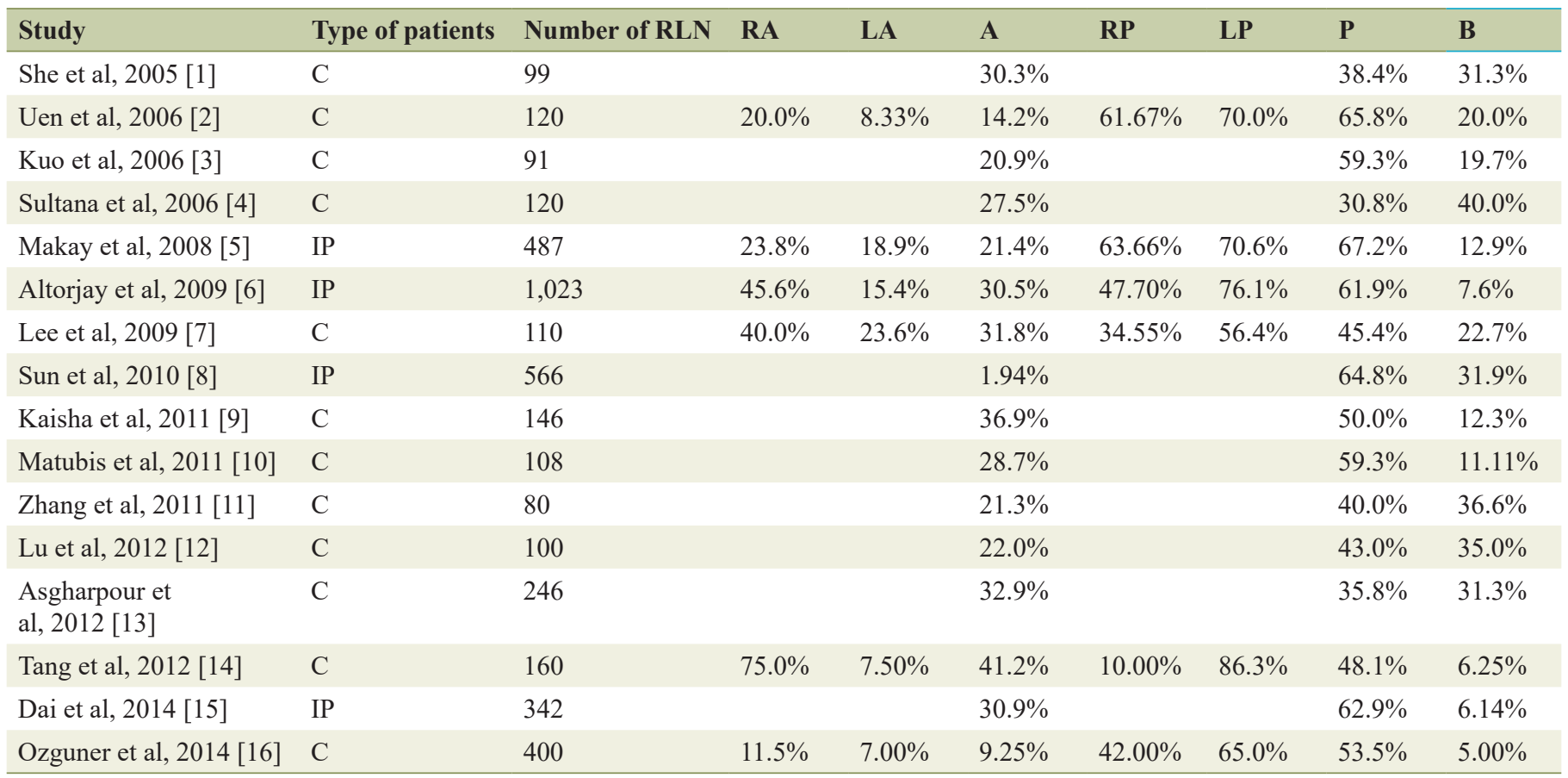

C: cadavers; IP: intraoperative finding; RLN: recurrent laryngeal nerve; ITA: inferior thyroid artery; RA: right anterior, LA: left anterior, A: anterior, RP: right posterior, LP: left posterior, P: posterior, B: between.

anterior to or between the ITA. Surgeons should also take care when performing operations of the thyroid on the right side because of the predominance of the anterior configuration [30].

There are studies suggesting that intraoperative nerve monitoring (IONM) has not proven more effective than direct nerve visualization has [31, 32]. However, when there is an anatomical variant, reoperation of the thyroid gland or obscured pathology IONM should be used to prevent iatrogenic injury of the RLN [33].

The purpose of our study is to highlight the variety in the normal anatomical course of RLNs during thyroidectomy to help surgeons perform safer operations and carry out accurate interventions to avoid lifelong complications in patients. In our study, after reviewing a total of 578 publications according to the PRISMA guidelines, we used 16 articles for both quantitative and qualitative analysis. After assessing 2,099 patients, we concluded that the RLN was usually running posterior to the
ITA, with a prevalence of $52.08 \%$. In addition, there were differences between the left and right sides of the thyroid gland, with the posterior type being more common on the left, representing an occurrence of $35.73 \%$.

Despite the large sample of patients included in our study and the statistical methods, which are the strength of our analysis, the heterogeneity was quite high according to the Higgins statistics. One explanation for this is the anatomical differences among races regarding the embryological development of the nervous and vascular systems. Another issue was that most of the studies used in the analysis were performed on cadavers and not during an operation. To exclude safe results with low statistical bias, it is necessary to conduct larger studies from the same continent in which surgery is performed on living subjects.

In conclusion, the relationship between the ITA and the RLN is highly variable. Thus, surgeons should always keep in mind the anatomical relationship of the RLN to the ITA and

Table 2. The Number of Studies Included, the Prevalence Estimate and the Statistical Heterogeneity According to Higgins $I^{2}$ Statistics

\begin{tabular}{|c|c|c|c|c|c|c|c|}
\hline Variables & $\begin{array}{l}\text { Number } \\
\text { of studies } \\
\text { (number } \\
\text { of nerves) }\end{array}$ & $\begin{array}{l}\text { Anterior \% } \\
(95 \% \text { CI) }\end{array}$ & $I^{2} \%(95 \%$ CI $)$ & $\begin{array}{l}\text { Posterior \% } \\
(95 \% \text { CI) }\end{array}$ & $I^{2} \%(95 \% C I)$ & $\begin{array}{l}\text { Between \% } \\
\text { (95\% CI) }\end{array}$ & $I^{2} \%(95 \%$ CI $)$ \\
\hline Right & $6(955)$ & $16.5(8.96-25.7)$ & $95,9(93.4-97.5)$ & $20.8(14.2-28.2)$ & $93.1(87.7-96.1)$ & & \\
\hline Left & $6(996)$ & $06.5(4.31-9.10)$ & $77.2(49.3-89.7)$ & $35.7(32.6-38.9)$ & $52.1(0-80.1)$ & & \\
\hline
\end{tabular}

$\mathrm{Cl}$ : confidence interval. 


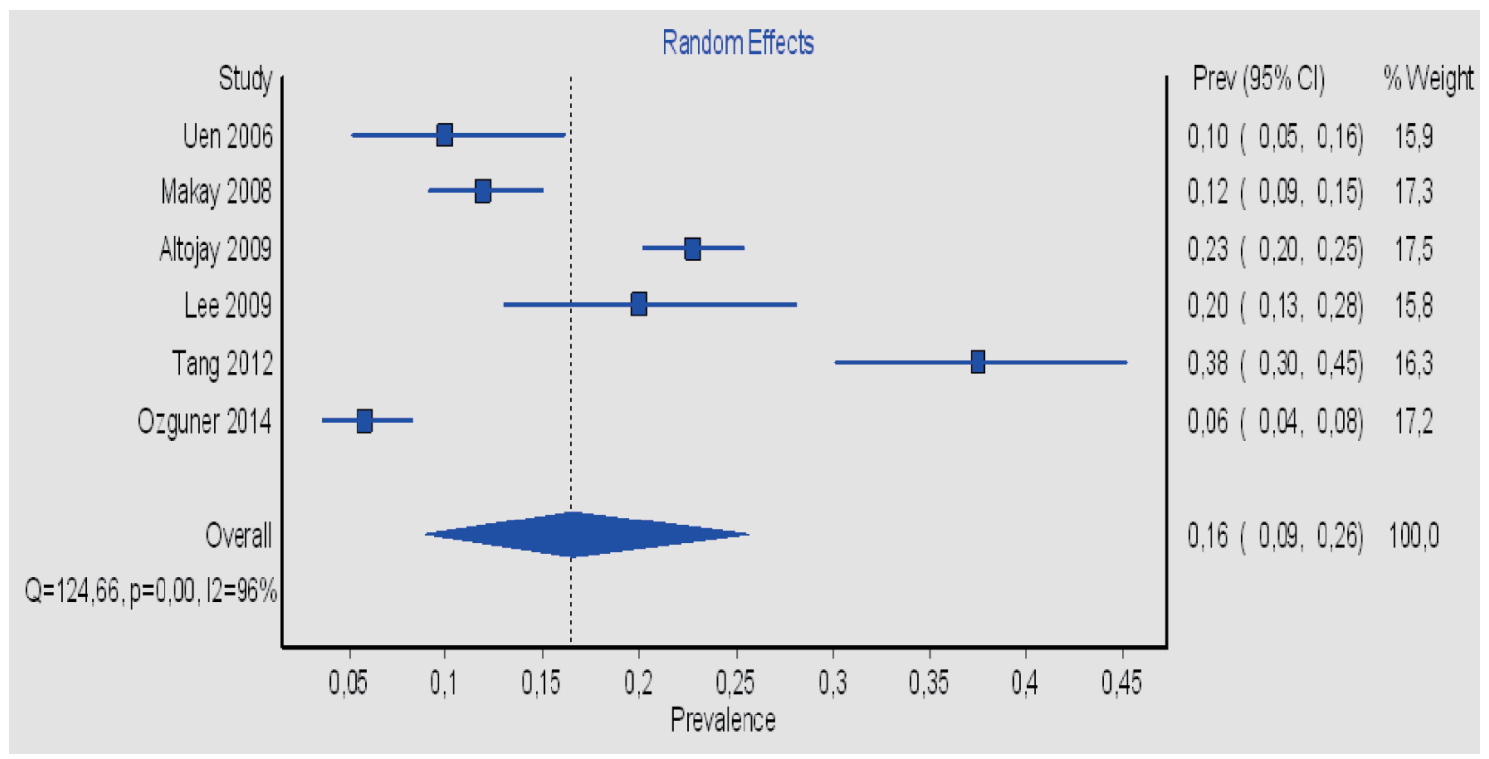

Figure 3. Forest plot for the right-sided prevalence of the RLN anterior to ITA. RLN: recurrent laryngeal nerve; ITA: inferior thyroid artery.

identify any abnormality to reduce morbidity and iatrogenic injury during thyroid surgery.

\section{Acknowledgments}

None to declare.

\section{Financial Disclosure}

There was no funding for this study.

\section{Conflict of Interest}

All authors declare that they have no conflict of interest.

\section{Author Contributions}

All authors contributed to the study conception and design. Material preparation, data collection and analysis were performed by Anastasios Katsourakis and George Karavasilis. The first draft of the manuscript was written by George Noussios and Anastasios Katsourakis, and all the authors commented on pre-

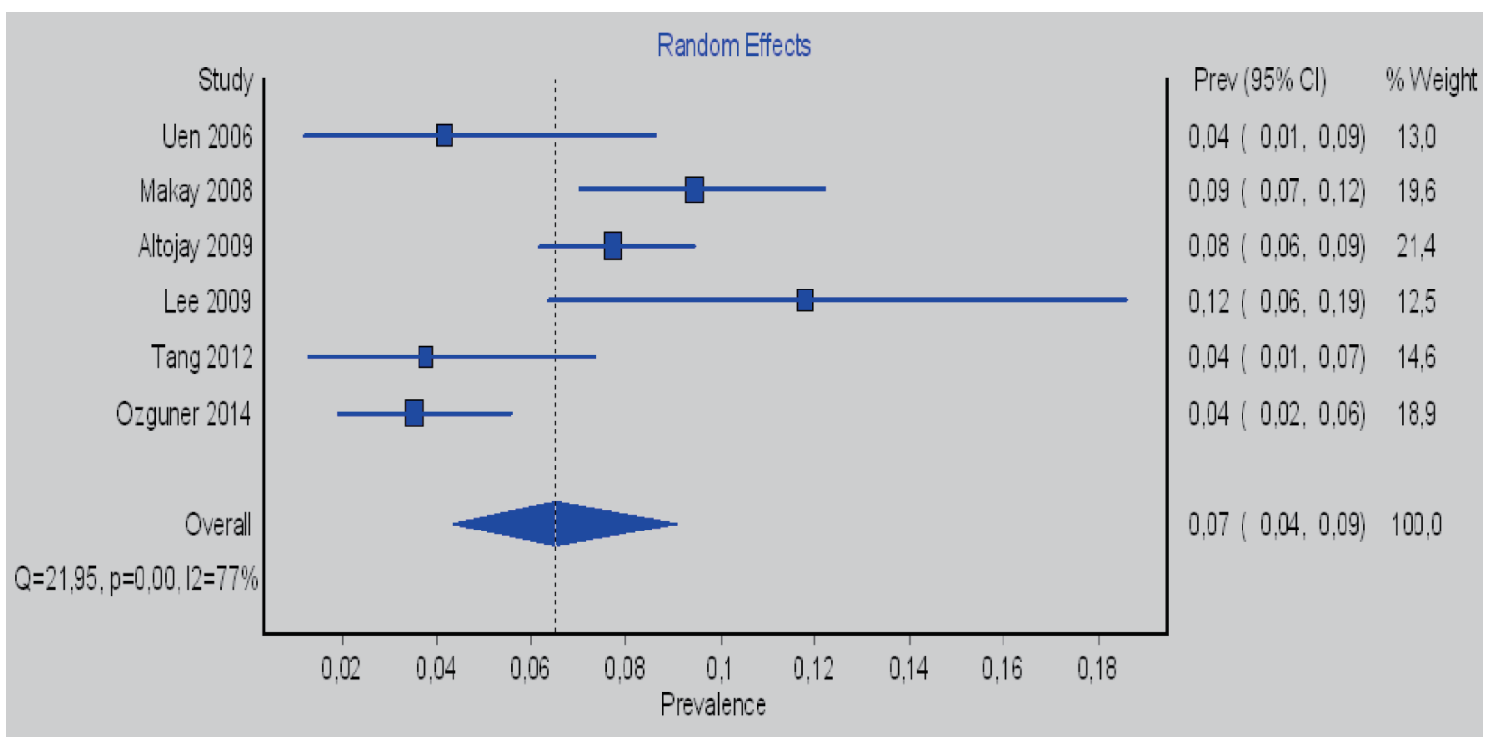

Figure 4. Forest plot for the left-sided prevalence of the RLN anterior to ITA. RLN: recurrent laryngeal nerve; ITA: inferior thyroid artery. 


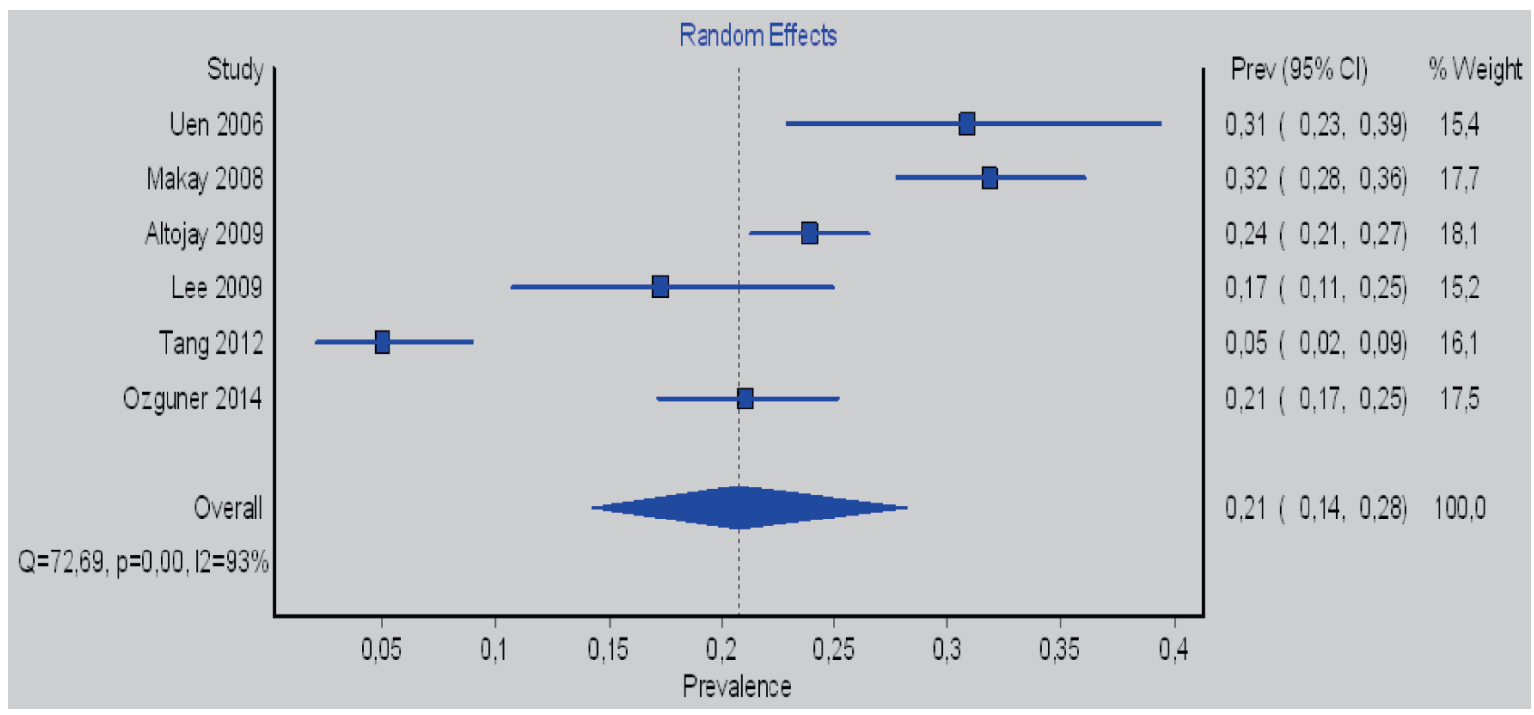

Figure 5. Forest plot for the right-sided prevalence of the RLN posterior to ITA. RLN: recurrent laryngeal nerve; ITA: inferior thyroid artery.

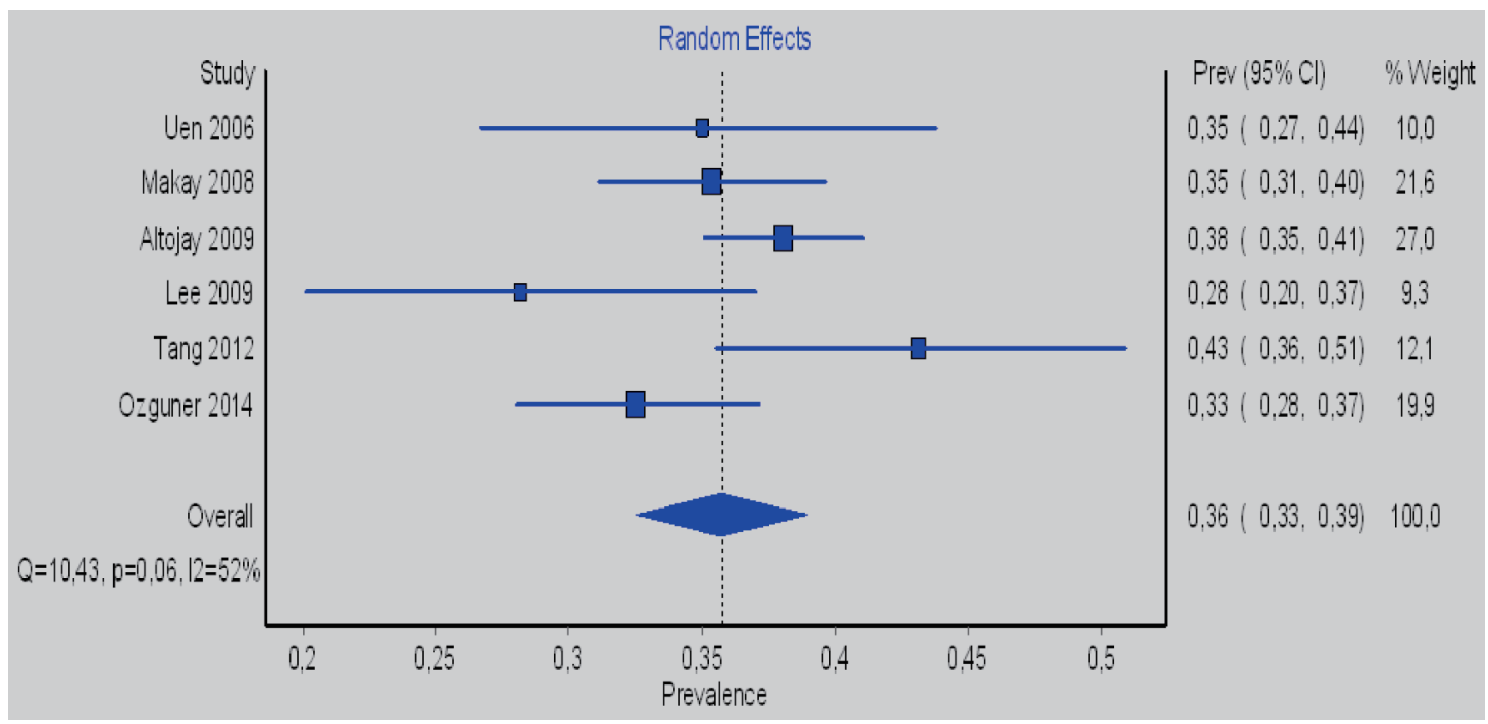

Figure 6. Forest plot for the left-sided prevalence of the RLN posterior to ITA. RLN: recurrent laryngeal nerve; ITA: inferior thyroid artery.

vious versions. All the authors approved the final manuscript.

\section{Data Availability}

The authors declare that data supporting the findings of this study are available within the article.

\section{References}

1. She Y, Zhang Z, Wu J, Qiuo F, Hu Y. Applied anatomy of the superior andrecurrent laryngeal nerves. J North Sichuan Med Coll. 2005;20:1-3.
2. Uen YH, Chen TH, Shyu JF, Shyr YM, Su CH, Chen JY, Lee CS, et al. Surgical anatomy of the recurrent laryngeal nerves and its clinical applications in chinese adults. Surg Today. 2006;36(4):312-315.

3. Kuo X, Yi D, Zhang W. Applied anatomy of the recurrent laryngeal nerve. Anat Res. 2006;28:222-224.

4. Sultana SZ, Khan MK, Rahman H, Hossain A, Sultana S, Hasan N, Mannan S, et al. Morphological study of recurrent laryngeal nerve in relation to thyroid gland. Mymensingh Med J. 2006;15(2):192-196.

5. Makay O, Icoz G, Yilmaz M, Akyildiz M, Yetkin E. The recurrent laryngeal nerve and the inferior thyroid artery anatomical variations during surgery. Langenbecks Arch Surg. 2008;393(5):681-685. 
6. Altorjay A, Tihanyi Z, Luka F, Juhasz A, Bencsik Z, Rull M, Kecskes G, et al. Place and value of the recurrent laryngeal nerve (RLN) palpatory method in preventing RLN palsy during thyroid surgery. Head Neck. 2009;31(4):538-547.

7. Lee MS, Lee UY, Lee JH, Han SH. Relative direction and position of recurrent laryngeal nerve for anatomical configuration. Surg Radiol Anat. 2009;31(9):649-655.

8. Sun M. Applied clinical anatomic study on recurrent laryngeal nerve. J Taishan Med Coll. 2010;31:679-681.

9. Kaisha W, Wobenjo A, Saidi H. Topography of the recurrent laryngeal nerve in relation to the thyroid artery, Zuckerkandl tubercle, and Berry ligament in Kenyans. Clin Anat. 2011;24(7):853-857.

10. Matubis JS, Dumlao KJP, Carrillo RJC. The recurrent laryngeal nerve in relation to the inferior thyroid artery in adult Filipino cadavers. Otolaryngol Head Neck Surg. 2011;1:13-17.

11. Zhang H, Cheng M. Anatomy of the cervical recurrent laryngeal nerve. Shandong Med J. 2011;51:110-111.

12. Lu Z, Zou J, Wan Y, Cao H, Xu W. Relationship between the recurrent laryngeal nerve and the inferior thyroid artery and its branches: an applied anatomical study. Chinese Arch Otolaryngol Neck Surg. 2012;19:548-551.

13. Asgharpour E, Maranillo E, Sanudo J, Pascual-Font A, Rodriguez-Niedenfuhr M, Valderrama FJ, Viejo F, et al. Recurrent laryngeal nerve landmarks revisited. Head Neck. 2012;34(9):1240-1246.

14. Tang WJ, Sun SQ, Wang XL, Sun YX, Huang HX. An applied anatomical study on the recurrent laryngeal nerve and inferior thyroid artery. Surg Radiol Anat. 2012;34(4):325-332.

15. Dai H, Hua Q, Jiang Y, Sheng J. [Anatomy of recurrent laryngeal nerve during thyroid surgery]. Lin Chung Er Bi Yan Hou Tou Jing Wai Ke Za Zhi. 2014;28(24):19251926, 1930.

16. Ozguner G, Sulak O. Arterial supply to the thyroid gland and the relationship between the recurrent laryngeal nerve and the inferior thyroid artery in human fetal cadavers. Clin Anat. 2014;27(8):1185-1192.

17. Giddings AE. The history of thyroidectomy. J R Soc Med. 1998;91(Suppl 33):3-6.

18. Sheikh NA, Khattak SF, Aleem A, Nadeem K. Diverse anatomical configuration of recurrent laryngeal nerve in relation to inferior thyroid artery, an experience with 51 thyroidectomies. J Ayub Med Coll Abbottabad. 2019;31(2):168-171.

19. Friedrich T, Steinert M, Keitel R, Sattler B, Schonfelder $\mathrm{M}$. [Incidence of damage to the recurrent laryngeal nerve in surgical therapy of various thyroid gland diseases-a retrospective study]. Zentralbl Chir. 1998;123(1):25-29.

20. Hayward NJ, Grodski S, Yeung M, Johnson WR, Serpell J. Recurrent laryngeal nerve injury in thyroid surgery: a review. ANZ J Surg. 2013;83(1-2):15-21.

21. Zakaria HM, Al Awad NA, Al Kreedes AS, Al-Mulhim AM, Al-Sharway MA, Hadi MA, Al Sayyah AA. Recurrent laryngeal nerve injury in thyroid surgery. Oman Med J. 2011;26(1):34-38

22. Jatzko GR, Lisborg PH, Muller MG, Wette VM. Recurrent nerve palsy after thyroid operations - principal nerve identification and a literature review. Surgery. 1994;115(2):139-144.

23. Ready AR, Barnes AD. Complications of thyroidectomy. Br J Surg. 1994;81(11):1555-1556.

24. Bergamaschi R, Becouarn G, Ronceray J, Arnaud JP. Morbidity of thyroid surgery. Am J Surg. 1998;176(1):71-75.

25. Wagner HE, Seiler C. Recurrent laryngeal nerve palsy after thyroid gland surgery. Br J Surg. 1994;81(2):226-228.

26. Rathi PK, Shaikh AR, Shaikh GA. Identification of recurrent laryngeal nerve during thyroidectomy decreases the risk of nerve injury. Pak J Med Sci. 2010;26(1):148-151.

27. Barczynski M, Konturek A, Pragacz K, Papier A, Stopa M, Nowak W. Intraoperative nerve monitoring can reduce prevalence of recurrent laryngeal nerve injury in thyroid reoperations: results of a retrospective cohort study. World J Surg. 2014;38(3):599-606.

28. Fowler C, Hanson W. Surgical anatomy of the thyroid gland with special reference to the relations of the recurrent laryngeal nerve. Surg Gynecol Obstet. 1929;49:59-66.

29. Skandalakis JE, Droulias C, Harlaftis N, Tzinas S, Gray SW, Akin JT, Jr. The recurrent laryngeal nerve. Am Surg. 1976;42(9):629-634.

30. Steurer M, Passler C, Denk DM, Schneider B, Niederle $\mathrm{B}$, Bigenzahn W. Advantages of recurrent laryngeal nerve identification in thyroidectomy and parathyroidectomy and the importance of preoperative and postoperative laryngoscopic examination in more than 1000 nerves at risk. Laryngoscope. 2002;112(1):124-133.

31. Chiang FY, Wang LF, Huang YF, Lee KW, Kuo WR. Recurrent laryngeal nerve palsy after thyroidectomy with routine identification of the recurrent laryngeal nerve. Surgery. 2005; 137(3):342-347.

32. Kasemsuwan L, Nubthuenetr S. Recurrent laryngeal nerve paralysis: a complication of thyroidectomy. J Otolaryngol. 1997;26(6):365-367.

33. Henry BM, Vikse J, Graves MJ, Sanna S, Sanna B, Tomaszewska IM, Hsieh WC, et al. Variable relationship of the recurrent laryngeal nerve to the inferior thyroid artery: A meta-analysis and surgical implications. Head Neck. 2017;39(1):177-186. 